\title{
Erratum
}

\section{Erratum to: Global Wellposed Problem for the 3-D Incompressible Anisotropic Navier-Stokes Equations in an Anisotropic Space}

\section{Ting Zhang}

Department of Mathematics, Zhejiang University, Hangzhou 310027, China.

E-mail: zhangting79@ hotmail.com

Received: 1 October 2009 / Accepted: 16 October 2009

Published online: 5 February 2010 - (C) Springer-Verlag 2010

Commun. Math Phys. 287, 211-224 (2009)

As it was pointed out by Ping Zhang, there is an error in the proof of Proposition 3.2 in our paper [1]. Hereby we would like to correct it and obtain Theorem 1.1 in [1] with the initial condition (1.4) being replaced by

$$
C_{1} v_{h}^{-1}\left\|u_{0}^{h}\right\|_{B^{0, \frac{1}{2}}} \exp \left\{C_{1}\left(v_{h}^{-1}\left\|u_{0}^{3}\right\|_{B^{0, \frac{1}{2}}}+1\right)^{8}\right\} \leq 1 .
$$

We apologize to the reader for this inconvenience.

At first, we obtain the following three lemmas.

Lemma 0.1. Let $u(t)$ and $\nabla_{h} u(t)$ be in $B^{0, \frac{1}{2}}$. We have

$$
\begin{aligned}
\left\|\Delta_{j}^{v}\left(u^{3} \partial_{h} u^{h}\right)(t)\right\|_{L_{h}^{\frac{4}{3}}\left(L_{v}^{2}\right)} \leq & C\left\|\nabla_{h} u^{3}\right\|_{B^{0, \frac{1}{2}}}^{\frac{1}{2}}\left[\left\|u^{3}\right\|_{B^{0, \frac{1}{2}}}^{\frac{1}{2}} \sum_{\left|j-j^{\prime}\right| \leq 5}\left\|\Delta_{j^{\prime}}^{v} \partial_{h} u^{h}\right\|_{L^{2}\left(\mathbb{R}^{3}\right)}\right. \\
& \left.+\sqrt{d_{j}(t)} \sqrt{d_{1 j}(t)} 2^{-\frac{j}{2}}\left\|\nabla_{h} u^{h}\right\|_{B^{0, \frac{1}{2}}}\left(\left\|u^{3}\right\|_{B^{0, \frac{1}{2}}}+\varepsilon_{1}\right)^{\frac{1}{2}}\right],
\end{aligned}
$$

where $\sum d_{j}(t) \leq 1, \varepsilon_{1}$ is any positive constant, $C$ is independent of $\varepsilon_{1}$, and

$$
d_{1 j}(t):=\sum_{j^{\prime} \geq j-N_{0}} \frac{2^{\frac{j}{2}}\left\|\Delta_{j^{\prime}}^{v} u^{3}\right\|_{L^{2}}}{\left\|u^{3}\right\|_{B^{0, \frac{1}{2}}}+\varepsilon_{1}},
$$

The online version of the original article can be found under doi:10.1007/s00220-008-0631-1. 
satisfying

$$
\sum_{j} d_{1 j} \leq C, \sum_{j} \sup _{t \in[0, T]} d_{1 j} \leq C \frac{\left\|u^{3}\right\|_{\widetilde{L}_{T}^{\infty}\left(B^{0, \frac{1}{2}}\right)}}{\varepsilon_{1}}
$$

Proof. Using Bony's decomposition in the vertical variable, we obtain

$$
\Delta_{j}^{v}\left(u^{3} \partial_{h} u^{h}\right)=\sum_{\left|j-j^{\prime}\right| \leq 5} \Delta_{j}^{v}\left(S_{j^{\prime}-1}^{v} u^{3} \partial_{h} \Delta_{j^{\prime}}^{v} u^{h}\right)+\sum_{j^{\prime} \geq j-N_{0}} \Delta_{j}^{v}\left(\Delta_{j^{\prime}}^{v} u^{3} \partial_{h} S_{j^{\prime}+2}^{v} u^{h}\right) .
$$

Using Hölder's inequality and Lemma 2.3 in [1], we get

$$
\begin{aligned}
\left\|\Delta_{j}^{v}\left(S_{j^{\prime}-1}^{v} u^{3} \partial_{h} \Delta_{j^{\prime}}^{v} u^{h}\right)\right\|_{L_{h}^{\frac{4}{3}\left(L_{v}^{2}\right)}} & \lesssim\left\|S_{j^{\prime}-1}^{v} u^{3}\right\|_{L_{h}^{4}\left(L_{v}^{\infty}\right)}\left\|\Delta_{j^{\prime}}^{v} \partial_{h} u^{h}\right\|_{L^{2}\left(\mathbb{R}^{3}\right)} \\
& \lesssim\left\|u^{3}\right\|_{B^{0, \frac{1}{2}}}^{\frac{1}{2}}\left\|\nabla_{h} u^{3}\right\|_{B^{0, \frac{1}{2}}}^{\frac{1}{2}}\left\|\Delta_{j^{\prime}}^{v} \partial_{h} u^{h}\right\|_{L^{2}\left(\mathbb{R}^{3}\right)}
\end{aligned}
$$

and

$$
\begin{aligned}
& \left\|\Delta_{j}^{v}\left(\Delta_{j^{\prime}}^{v} u^{3} \partial_{h} S_{j^{\prime}+2}^{v} u^{h}\right)(t)\right\|_{L_{h}^{\frac{4}{3}}\left(L_{v}^{2}\right)} \\
& \quad \lesssim\left\|S_{j^{\prime}+2}^{v}\left(\partial_{h} u^{h}\right)(t)\right\|_{L_{h}^{2}\left(L_{v}^{\infty}\right)}\left\|\Delta_{j^{\prime}}^{v} u^{3}(t)\right\|_{L_{h}^{4}\left(L_{v}^{2}\right)} \\
& \quad \lesssim\left\|\nabla_{h} u^{h}\right\|_{B^{0, \frac{1}{2}}}\left(\frac{2^{\frac{j^{\prime}}{2}}\left\|\Delta_{j^{\prime}}^{v} u^{3}\right\|_{L^{2}}}{\left\|u^{3}\right\|_{B^{0, \frac{1}{2}}}+\varepsilon_{1}}\right)^{\frac{1}{2}} 2^{-\frac{j^{\prime}}{4}}\left(\left\|u^{3}\right\|_{B^{0, \frac{1}{2}}}+\varepsilon_{1}\right)^{\frac{1}{2}} \sqrt{d_{j^{\prime}}(t)} 2^{-\frac{j^{\prime}}{4}}\left\|\nabla_{h} u^{3}\right\|_{B^{0, \frac{1}{2}}}^{\frac{1}{2}} .
\end{aligned}
$$

Then, we can immediately finish the proof.

Lemma 0.2. Let $u$ be in $B^{0, \frac{1}{2}}(T)$. We have

$$
\begin{aligned}
& \left\|\Delta_{j}^{v}\left(u^{3} u^{h}\right)\right\|_{L_{T}^{2}\left(L^{2}\left(\mathbb{R}^{3}\right)\right)} \\
& \leq C 2^{-\frac{j}{2}} \sqrt{d_{j}}\left\|\nabla_{h} u^{h}\right\|_{\widetilde{L}_{T}^{2}\left(B^{\left.0, \frac{1}{2}\right)}\right.}^{\frac{1}{2}}\left\|d_{2 j}(t)\right\| \nabla_{h} u^{3}\left\|_{B^{0, \frac{1}{2}}}\right\| u^{3}\left\|_{B^{0, \frac{1}{2}}}\left(\left\|u^{h}\right\|_{B^{0, \frac{1}{2}}}+\varepsilon_{2}\right)\right\|_{L_{T}^{2}}^{\frac{1}{2}} \\
& \quad+C 2^{-\frac{j}{2}}\left\|\sqrt{d_{j}(t)}\right\| \nabla_{h} u^{3}\left\|_{B^{0, \frac{1}{2}}}^{\frac{1}{2}}\right\| \nabla_{h} u^{h}\left\|_{B^{0, \frac{1}{2}}}^{\frac{1}{2}} \sqrt{d_{1 j}(t)}\right\| u^{h}\left\|_{B^{0, \frac{1}{2}}}^{\frac{1}{2}}\left(\left\|u^{3}\right\|_{B^{0, \frac{1}{2}}}+\varepsilon_{1}\right)^{\frac{1}{2}}\right\|_{L_{T}^{2}},
\end{aligned}
$$

where $\sum d_{j} \leq 1, \varepsilon_{1}$ and $\varepsilon_{2}$ are any positive constants, $C$ is independent of $\varepsilon_{1}$ and $\varepsilon_{2}$, $d_{1 j}(t)$ is defined in Lemma 0.1 and

$$
d_{2 j}(t):=\sum_{\left|j-j^{\prime}\right| \leq 5} \frac{2^{\frac{j^{\prime}}{2}}\left\|\Delta_{j^{\prime}}^{v} u^{h}\right\|_{L^{2}}}{\left\|u^{h}\right\|_{B^{0, \frac{1}{2}}}+\varepsilon_{2}}, \sum_{j} \sup _{t \in[0, T]} d_{2 j} \leq C \frac{\left\|u^{h}\right\|_{\widetilde{L}_{T}^{\infty}\left(B^{0, \frac{1}{2}}\right)}}{\varepsilon_{2}} .
$$

Proof. Using Bony's decomposition in the vertical variable, we obtain

$$
\Delta_{j}^{v}\left(u^{3} u^{h}\right)=\sum_{\left|j-j^{\prime}\right| \leq 5} \Delta_{j}^{v}\left(S_{j^{\prime}-1}^{v} u^{3} \Delta_{j^{\prime}}^{v} u^{h}\right)+\sum_{j^{\prime} \geq j-N_{0}} \Delta_{j}^{v}\left(S_{j^{\prime}+2}^{v} u^{h} \Delta_{j^{\prime}}^{v} u^{3}\right) .
$$


Using Hölder's inequality and Lemma 2.3 in [1], we get

$$
\begin{aligned}
& \left\|\Delta_{j}^{v}\left(S_{j^{\prime}-1}^{v} u^{3} \Delta_{j^{\prime}}^{v} u^{h}\right)\right\|_{L_{T}^{2}\left(L^{2}\left(\mathbb{R}^{3}\right)\right)} \\
& \lesssim\|\| S_{j^{\prime}-1}^{v} u^{3}\left\|_{L_{v}^{\infty}\left(L_{h}^{4}\right)}\right\| \Delta_{j^{\prime}}^{v} u^{h}\left\|_{L_{v}^{2}\left(L_{h}^{4}\right)}\right\|_{L_{T}^{2}} \\
& \lesssim\left\|u^{3}\right\|_{B^{0, \frac{1}{2}}}^{\frac{1}{2}}\left\|\nabla_{h} u^{3}\right\|_{B^{0, \frac{1}{2}}}^{\frac{1}{2}}\left(\frac{2^{\frac{j^{\prime}}{2}}\left\|\Delta_{j^{\prime}}^{v} u^{h}\right\|_{L^{2}}}{\left\|u^{h}\right\|_{B^{0, \frac{1}{2}}}+\varepsilon_{2}}\right)^{\frac{1}{2}} \\
& \times 2^{-\frac{j^{\prime}}{4}}\left(\left\|u^{h}\right\|_{B^{0, \frac{1}{2}}}+\varepsilon_{2}\right)^{\frac{1}{2}}\left\|\nabla_{h} \Delta_{j^{\prime}}^{v} u^{h}\right\|_{L^{2}}^{\frac{1}{2}} \|_{L_{T}^{2}} \\
& \lesssim\left\|u_{B^{0, \frac{1}{2}}}^{\frac{1}{2}}\right\| \nabla_{h} u^{3}\left\|_{B^{0, \frac{1}{2}}}^{\frac{1}{2}}\left(\frac{2^{\frac{j^{\prime}}{2}}\left\|\Delta_{j^{\prime}}^{v} u^{h}\right\|_{L^{2}}}{\left\|u^{h}\right\|_{B^{0, \frac{1}{2}}}+\varepsilon_{2}}\right)^{\frac{1}{2}} 2^{-\frac{j^{\prime}}{4}}\left(\left\|u^{h}\right\|_{B^{0, \frac{1}{2}}}+\varepsilon_{2}\right)^{\frac{1}{2}}\right\|_{L_{T}^{4}} \\
& \times d_{j^{\prime}}^{\frac{1}{2}} 2^{-\frac{j^{\prime}}{4}}\left\|\nabla_{h} u^{h}\right\|_{\widetilde{L}_{T}^{2}\left(B^{0, \frac{1}{2}}\right)}^{\frac{1}{2}}
\end{aligned}
$$

and

$$
\begin{aligned}
& \left\|\Delta_{j}^{v}\left(S_{j^{\prime}+2}^{v} u^{h} \Delta_{j^{\prime}}^{v} u^{3}\right)(t)\right\|_{L^{2}\left(\mathbb{R}^{3}\right)} \\
& \lesssim\left\|S_{j^{\prime}+2}^{v} u^{h}\right\|_{L_{v}^{\infty}\left(L_{h}^{4}\right)}\left\|\Delta_{j^{\prime}}^{v} u^{3}\right\|_{L_{v}^{2}\left(L_{h}^{4}\right)} \\
& \lesssim\left\|u^{h}\right\|_{B^{0, \frac{1}{2}}}^{\frac{1}{2}}\left\|\nabla_{h} u^{h}\right\|_{B^{0, \frac{1}{2}}}^{\frac{1}{2}}\left(\frac{2^{\frac{j^{\prime}}{2}}\left\|\Delta_{j^{\prime}}^{v} u^{3}\right\|_{L^{2}}}{\left\|u^{3}\right\|_{B^{0, \frac{1}{2}}}+\varepsilon_{1}}\right)^{\frac{1}{2}} \\
& \quad \times 2^{-\frac{j^{\prime}}{4}}\left(\left\|u^{3}\right\|_{B^{0, \frac{1}{2}}}+\varepsilon_{1}\right)^{\frac{1}{2}} \sqrt{d_{j^{\prime}}(t)} 2^{-\frac{j^{\prime}}{4}}\left\|\nabla_{h} u^{3}\right\|_{B^{0, \frac{1}{2}}}^{\frac{1}{2}} .
\end{aligned}
$$

Then, we can immediately finish the proof.

Similarly, one can obtain the following lemma.

Lemma 0.3. Let $u$ and $w$ be in $B^{0, \frac{1}{2}}(T)$. We have

$$
\left\|\Delta_{j}^{v}(u w)\right\|_{L_{T}^{2}\left(L^{2}\left(\mathbb{R}^{3}\right)\right)} \lesssim d_{j} v_{h}^{-\frac{1}{2}} 2^{-\frac{j}{2}}\|u\|_{B^{0, \frac{1}{2}}(T)}\|w\|_{B^{0, \frac{1}{2}}(T)} .
$$

Then, we can correct Proposition 3.2 in [1] as follows.

Proposition 0.1. Let $u$ be a divergence free vector filed in $B^{0, \frac{1}{2}}(T)$. Then, for any $j \in \mathbb{Z}$, we have 


$$
\begin{aligned}
& G_{j}(T):=\int_{0}^{T}\left|\sum_{k, l} \int_{\mathbb{R}^{3}} \Delta_{j}^{v}(-\Delta)^{-1} \partial_{l} \partial_{k}\left(u^{l} u^{k}\right) \Delta_{j}^{v} \partial_{h} w d x\right| d t \\
& \leq C d_{j}^{2} v_{h}^{-1} 2^{-j}\left\|u^{h}\right\|_{B^{0, \frac{1}{2}}(T)}^{3}+C 2^{-\frac{j}{2}}\left\|\Delta_{j}^{v} \partial_{h} w\right\|_{L_{T}^{2}\left(L^{2}\right)} \\
& \times\left[d_{j}^{\frac{1}{2}}\left\|\nabla_{h} u^{h}\right\|_{\widetilde{L}_{T}^{2}\left(B^{0, \frac{1}{2}}\right)}^{\frac{1}{2}}\left\|d_{2 j}(t)\right\| \nabla_{h} u^{3}\left\|_{B^{0, \frac{1}{2}}}\right\| u^{3}\left\|_{B^{0, \frac{1}{2}}}\left(\left\|u^{h}\right\|_{B^{0, \frac{1}{2}}}+\varepsilon_{2}\right)\right\|_{L_{T}^{2}}^{\frac{1}{2}}\right. \\
& \left.+\left\|\sqrt{d_{j}(t)}\right\| \nabla_{h} u^{3}\left\|_{B^{0, \frac{1}{2}}}^{\frac{1}{2}}\right\| \nabla_{h} u^{h}\left\|_{B^{0, \frac{1}{2}}}^{\frac{1}{2}} \sqrt{d_{1 j}(t)}\right\| u^{h}\left\|_{B^{0, \frac{1}{2}}}^{\frac{1}{2}}\left(\left\|u^{3}\right\|_{B^{0, \frac{1}{2}}}+\varepsilon_{1}\right)^{\frac{1}{2}}\right\|_{L_{T}^{2}}\right] \\
& +C \int_{0}^{T}\left\|\nabla_{h} u^{3}\right\|_{B^{0, \frac{1}{2}}}^{\frac{1}{2}}\left[\sqrt{d_{j}(t)} \sqrt{d_{1 j}(t)} 2^{-\frac{j}{2}}\left\|\nabla_{h} u^{h}\right\|_{B^{0, \frac{1}{2}}}\left(\left\|u^{3}\right\|_{B^{0, \frac{1}{2}}}+\varepsilon_{1}\right)^{\frac{1}{2}}\right. \\
& \left.+\left\|u^{3}\right\|_{B^{0, \frac{1}{2}}}^{\frac{1}{2}} \sum_{\left|j-j^{\prime}\right| \leq 5}\left\|\Delta_{j^{\prime}}^{v} \partial_{h} u^{h}\right\|_{L^{2}\left(\mathbb{R}^{3}\right)}\right] \sqrt{d_{3 j}(t)} 2^{-\frac{j}{4}}\left(\left\|u^{h}\right\|_{B^{0, \frac{1}{2}}}+\varepsilon_{2}\right)^{\frac{1}{2}}\left\|\Delta_{j}^{v} \nabla_{h} w\right\|_{L^{2}}^{\frac{1}{2}} d t,
\end{aligned}
$$

where $w=u^{1}$ or $u^{2}, C$ is independent of $\varepsilon_{1}$ and $\varepsilon_{2}$, and

$$
d_{3 j}(t):=\frac{2^{\frac{j}{2}}\left\|\Delta_{j}^{v} u^{h}\right\|_{L^{2}}}{\left\|u^{h}\right\|_{B^{0, \frac{1}{2}}}+\varepsilon_{2}}, \sum_{j} \sup _{t \in[0, T]} d_{3 j} \leq \frac{\left\|u^{h}\right\|_{\widetilde{L}_{T}^{\infty}\left(B^{0, \frac{1}{2}}\right)}}{\varepsilon_{2}} .
$$

Proof. We distinguish the terms with horizontal derivatives from the terms with vertical ones, writing

$$
G_{j}(T) \leq G_{j}^{h}(T)+2 G_{j}^{v 1}(T)+G_{j}^{v 2}(T),
$$

where

$$
\begin{aligned}
G_{j}^{h}(T) & :=\sum_{l=1}^{2} \sum_{k=1}^{2} \int_{0}^{T}\left|\int_{\mathbb{R}^{3}} \Delta_{j}^{v}(-\Delta)^{-1} \partial_{l} \partial_{k}\left(u^{l} u^{k}\right) \Delta_{j}^{v} \partial_{h} w d x\right| d t, \\
G_{j}^{v 1}(T) & :=\sum_{k=1}^{2} \int_{0}^{T}\left|\int_{\mathbb{R}^{3}} \Delta_{j}^{v}(-\Delta)^{-1} \partial_{3} \partial_{k}\left(u^{3} u^{k}\right) \Delta_{j}^{v} \partial_{h} w d x\right| d t,
\end{aligned}
$$

and

$$
G_{j}^{v 2}(T):=\int_{0}^{T}\left|\int_{\mathbb{R}^{3}} \Delta_{j}^{v}(-\Delta)^{-1} \partial_{3}\left(2 u^{3} \partial_{3} u^{3}\right) \Delta_{j}^{v} \partial_{h} w d x\right| d t .
$$

Using Hölder's inequality and Lemma 0.3 , we get

$$
\begin{aligned}
G_{j}^{h}(T) & \lesssim \sum_{l=1}^{2} \sum_{k=1}^{2}\left\|\Delta_{j}^{v}\left(u^{l} u^{k}\right)\right\|_{L_{T}^{2}\left(L^{2}\right)}\left\|\Delta_{j}^{v} \partial_{h} u^{h}\right\|_{L_{T}^{2}\left(L^{2}\right)} \\
& \lesssim d_{j} v_{h}^{-\frac{1}{2}} 2^{-\frac{j}{2}}\left\|u^{h}\right\|_{B^{0, \frac{1}{2}}(T)}^{2}\left\|\Delta_{j}^{v} \partial_{h} u^{h}\right\|_{L_{T}^{2}\left(L^{2}\right)} \\
& \lesssim d_{j}^{2} v_{h}^{-1} 2^{-j}\left\|u^{h}\right\|_{B}^{3, \frac{1}{2}(T)} .
\end{aligned}
$$


Similarly, using Hölder's inequality and Lemma 0.2, we have

$$
\begin{aligned}
& G_{j}^{v 1}(T) \\
& \lesssim \sum_{k=1}^{2}\left\|\Delta_{j}^{v}\left(u^{3} u^{k}\right)\right\|_{L_{T}^{2}\left(L^{2}\right)}\left\|\Delta_{j}^{v} \partial_{h} w\right\|_{L_{T}^{2}\left(L^{2}\right)} \\
& \lesssim 2^{-\frac{j}{2}}\left\|\Delta_{j}^{v} \partial_{h} w\right\|_{L_{T}^{2}\left(L^{2}\right)}\left[d_{j}^{\frac{1}{2}}\left\|\nabla_{h} u^{h}\right\|_{\widetilde{L}_{T}^{2}\left(B^{0, \frac{1}{2}}\right)}^{\frac{1}{2}}\left\|d_{2 j}(t)\right\| \nabla_{h} u^{3}\left\|_{B^{0, \frac{1}{2}}}\right\| u^{3}\left\|_{B^{0, \frac{1}{2}}}\left(\left\|u^{h}\right\|_{B^{0, \frac{1}{2}}}+\varepsilon_{2}\right)\right\|_{L_{T}^{2}}^{\frac{1}{2}}\right. \\
& +\| \sqrt{\left.d_{j}(t)\left\|\nabla_{h} u^{3}\right\|_{B^{0, \frac{1}{2}}}^{\frac{1}{2}}\left\|\nabla_{h} u^{h}\right\|_{B^{0, \frac{1}{2}}}^{\frac{1}{2}} \sqrt{d_{1 j}(t)}\left\|u^{h}\right\|_{B^{0, \frac{1}{2}}}^{\frac{1}{2}}\left(\left\|u^{3}\right\|_{B^{0, \frac{1}{2}}}+\varepsilon_{1}\right)^{\frac{1}{2}} \|_{L_{T}^{2}}\right] .}
\end{aligned}
$$

Since $\operatorname{div} u=0$, we obtain

$$
\begin{aligned}
G_{j}^{v 2}(T) & =\int_{0}^{T}\left|\int_{\mathbb{R}^{3}} \Delta_{j}^{v}(-\Delta)^{-1} \partial_{3}\left(2 u^{3} \operatorname{div}_{h} u^{h}\right) \Delta_{j}^{v} \partial_{h} w d x\right| d t \\
& =\int_{0}^{T}\left|\int_{\mathbb{R}^{3}} \Delta_{j}^{v}\left(2 u^{3} \operatorname{div}_{h} u^{h}\right) \Delta_{j}^{v}(-\Delta)^{-1} \partial_{3} \partial_{h} w d x\right| d t .
\end{aligned}
$$

Then, using Hölder's inequality, Minkowski’s inequality, Lemma 2.3 in [1] and Lemma 0.1 , we get

$$
\begin{aligned}
G_{j}^{v 2}(T) \lesssim & \int_{0}^{T}\left\|\Delta_{j}^{v}\left(u^{3} \operatorname{div}_{h} u^{h}\right)\right\|_{L_{h}^{\frac{4}{3}}\left(L_{v}^{2}\right)}\left\|\Delta_{j}^{v}(-\Delta)^{-1} \partial_{h} \partial_{3} w\right\|_{L_{h}^{4}\left(L_{v}^{2}\right)} d t \\
\lesssim & \int_{0}^{T}\left\|\nabla_{h} u^{3}\right\|_{B^{0, \frac{1}{2}}}^{\frac{1}{2}}\left[\sqrt{d_{j}(t)} \sqrt{d_{1 j}(t)} 2^{-\frac{j}{2}}\left\|\nabla_{h} u^{h}\right\|_{B^{0, \frac{1}{2}}}\left(\left\|u^{3}\right\|_{B^{0, \frac{1}{2}}}+\varepsilon_{1}\right)^{\frac{1}{2}}\right. \\
& \left.+\left\|u^{3}\right\|_{B^{0, \frac{1}{2}}}^{\frac{1}{2}} \sum_{\left|j-j^{\prime}\right| \leq 5}\left\|\Delta_{j^{\prime}}^{v} \partial_{h} u^{h}\right\|_{L^{2}\left(\mathbb{R}^{3}\right)}\right]\left\|\Delta_{j}^{v} w\right\|_{L^{2}}^{\frac{1}{2}}\left\|\Delta_{j}^{v} \nabla_{h} w\right\|_{L^{2}}^{\frac{1}{2}} d t .
\end{aligned}
$$

This completes the proof of Proposition 0.1.

Similarly, we can obtain the following proposition and omit the details.

Proposition 0.2. Let $u$ be a divergence free vector field in $B^{0, \frac{1}{2}}(T)$. Then, for any $j \in \mathbb{Z}$, we have

$$
\begin{aligned}
G_{j}(T) & :=\int_{0}^{T}\left|\sum_{k, l} \int_{\mathbb{R}^{3}} \Delta_{j}^{v}(-\Delta)^{-1} \partial_{l} \partial_{k}\left(u^{l} u^{k}\right) \Delta_{j}^{v} \partial_{h} u^{h} d x\right| d t \\
& \lesssim d_{j}^{2} v_{h}^{-1} 2^{-j}\left[\left\|u^{h}\right\|_{B^{0, \frac{1}{2}}(T)}^{3}+\left\|u^{h}\right\|_{B^{0, \frac{1}{2}}(T)}^{2}\left\|u^{3}\right\|_{B^{0, \frac{1}{2}}(T)}\right] .
\end{aligned}
$$

Proof of the existence part of Theorem 1.1 in [1] with the initial condition (0.1). Applying the operator $\Delta_{j}^{v}$ to (3.1) in [1] and taking the $L^{2}$ inner product of the resulting 
882

T. Chang

equation with $\Delta_{j}^{v} u_{n}^{1}$, from Proposition 3.1 in [1], Proposition 0.1 and the CauchySchwartz inequality, we get

$$
\begin{aligned}
2^{j} & \left(\left\|\Delta_{j}^{v} u_{n}^{1}(t)\right\|_{L^{2}}^{2}+v_{h}\left\|\nabla_{h} \Delta_{j}^{v} u_{n}^{1}\right\|_{L_{t}^{2}\left(L^{2}\right)}^{2}+2 v_{3}\left\|\partial_{3} \Delta_{j}^{v} u_{n}^{1}\right\|_{L_{t}^{2}\left(L^{2}\right)}^{2}\right) \\
\leq & 2^{j}\left\|\Delta_{j}^{v} u_{n}^{1}(0)\right\|_{L^{2}}^{2}+C d_{j}^{2} v_{h}^{-1}\left\|u_{n}^{h}\right\|_{B^{0, \frac{1}{2}}(t)}^{3} \\
& +C v_{h}^{-1} d_{j}\left\|\nabla_{h} u^{h}\right\|_{\widetilde{L}_{t}^{2}\left(B^{0, \frac{1}{2}}\right)}\left(\int_{0}^{t} d_{2 j}^{2}(s)\left\|\nabla_{h} u^{3}\right\|_{B^{0, \frac{1}{2}}}^{2}\left\|u^{3}\right\|_{B^{0, \frac{1}{2}}}^{2}\left(\left\|u^{h}\right\|_{B^{0, \frac{1}{2}}}+\varepsilon_{2}\right)^{2} d s\right)^{\frac{1}{2}} \\
& +C v_{h}^{-1} \int_{0}^{t} d_{j}(s)\left\|\nabla_{h} u^{3}\right\|_{B^{0, \frac{1}{2}}}\left\|\nabla_{h} u^{h}\right\|_{B^{0, \frac{1}{2}}} d_{1 j}(s)\left\|u^{h}\right\|_{B^{0, \frac{1}{2}}}\left(\left\|u^{3}\right\|_{B^{0, \frac{1}{2}}}+\varepsilon_{1}\right) d s \\
& +C v_{h}^{-\frac{1}{3}} \int_{0}^{t}\left\|\nabla_{h} u^{3}\right\|_{B^{0, \frac{1}{2}}}^{\frac{2}{3}} d_{j}^{\frac{2}{3}}(s) d_{1 j}^{\frac{2}{3}}(s)\left\|\nabla_{h} u^{h}\right\|_{B^{0, \frac{1}{2}}}^{\frac{4}{3}}\left(\left\|u^{3}\right\|_{B^{0, \frac{1}{2}}}+\varepsilon_{1}\right)^{\frac{2}{3}} d_{3 j}^{\frac{2}{3}}(s) \\
& \times\left(\left\|u^{h}\right\|_{B^{0, \frac{1}{2}}}+\varepsilon_{2}\right)^{\frac{2}{3}} d s \\
& +C v_{h}^{-\frac{1}{3}}\left(\int_{0}^{t} d_{3 j}^{2}(t)\left\|\nabla_{h} u^{3}\right\|_{B^{0, \frac{1}{2}}}^{2}\left\|u^{3}\right\|_{B^{0, \frac{1}{2}}}^{2}\left(\left\|u^{h}\right\|_{B^{0, \frac{1}{2}}}+\varepsilon_{2}\right)^{2} d s\right)^{\frac{1}{3}} \\
& \times\left(\sum_{\left|j-j^{\prime}\right| \leq 5} 2^{j^{\prime}}\left\|\Delta_{j^{\prime}}^{v} \partial_{h} u^{h}\right\|_{L_{t}^{2}\left(L^{2}\left(\mathbb{R}^{3}\right)\right)}^{2}\right)^{\frac{2}{3}}
\end{aligned}
$$

and

$$
\begin{aligned}
& \left\|u_{n}^{1}(t)\right\|_{B^{0, \frac{1}{2}}}+\sqrt{v_{h}}\left\|\nabla_{h} u_{n}^{1}\right\|_{\widetilde{L}_{t}^{2}\left(B^{0, \frac{1}{2}}\right)}+\sqrt{\nu_{3}}\left\|\partial_{3} \Delta_{j}^{v} u_{n}^{1}\right\|_{\widetilde{L}_{t}^{2}\left(B^{0, \frac{1}{2}}\right)} \\
& \leq C\left\|u_{n}^{1}(0)\right\|_{B^{0, \frac{1}{2}}}+C v_{h}^{-\frac{1}{2}}\left\|u_{n}^{h}\right\|_{B^{0, \frac{1}{2}}(t)}^{\frac{3}{2}} \\
& \quad+C v_{h}^{-\frac{1}{2}}\left\|\nabla_{h} u^{h}\right\|_{\widetilde{L}_{t}^{2}\left(B^{0, \frac{1}{2}}\right)}^{\frac{1}{2}}\|\| \nabla_{h} u^{3}\left\|_{B^{0, \frac{1}{2}}}\right\| u^{3}\left\|_{B^{0, \frac{1}{2}}}\left(\left\|u^{h}\right\|_{B^{0, \frac{1}{2}}}+\varepsilon_{2}\right)\right\|_{L_{t}^{2}}^{\frac{1}{2}}\left\|\sup _{s \in[0, t]} d_{2 j}(s)\right\|_{l_{j}^{1}}^{\frac{1}{4}} \\
& \quad+C v_{h}^{-\frac{1}{2}}\left(\int_{0}^{t}\left\|\nabla_{h} u^{h}\right\|_{B^{0, \frac{1}{2}}}\left\|\nabla_{h} u^{3}\right\|_{B^{0, \frac{1}{2}}}\left\|u^{h}\right\|_{B^{0, \frac{1}{2}}}\left(\left\|u^{3}\right\|_{B^{0, \frac{1}{2}}}+\varepsilon_{1}\right) d s\right)^{\frac{1}{2}}\left\|\sup _{s \in[0, t]} d_{1 j}(s)\right\|_{l_{j}^{1}}^{\frac{1}{2}} \\
& \quad+C v_{h}^{-\frac{1}{6}}\left(\int_{0}^{t}\left\|\nabla_{h} u^{3}\right\|_{B^{0, \frac{1}{2}}}^{\frac{2}{3}}\left\|\nabla_{h} u^{h}\right\|_{B^{0, \frac{1}{2}}}^{\frac{4}{3}}\left(\left\|u^{3}\right\|_{B^{0, \frac{1}{2}}}+\varepsilon_{1}\right)^{\frac{2}{3}}\left(\left\|u^{h}\right\|_{B^{0, \frac{1}{2}}}+\varepsilon_{2}\right)^{\frac{2}{3}} d s\right)^{\frac{1}{2}} \\
& \quad \times\left\|\sup _{s \in[0, t]} d_{1 j}^{\frac{2}{3}}(s) d_{3_{j}^{3}}^{\frac{1}{3}}(s)\right\|_{l_{j}^{1}}^{\frac{1}{2}} \\
& \quad+C v_{h}^{-\frac{3}{2}}\left(\int_{0}^{t}\left\|\nabla_{h} u^{3}\right\|_{B^{0, \frac{1}{2}}}^{2}\left\|u^{3}\right\|_{B^{0, \frac{1}{2}}}^{2}\left(\left\|u^{h}\right\|_{B^{0, \frac{1}{2}}}+\varepsilon_{2}\right)^{2} d s\right)^{\frac{1}{2}}\left\|\sup _{s \in[0, t]} d_{3 j}(s)\right\|_{l_{j}^{1}}^{\frac{1}{2}}
\end{aligned}
$$




$$
\begin{aligned}
\leq & C\left\|u_{n}^{1}(0)\right\|_{B^{0, \frac{1}{2}}}+C v_{h}^{-\frac{1}{2}}\left\|u_{n}^{h}\right\|_{B^{0, \frac{1}{2}}(T)}^{\frac{3}{2}}+\frac{\sqrt{v_{h}}}{4}\left\|\nabla_{h} u_{n}^{h}\right\|_{\widetilde{L}_{t}^{2}\left(B^{0, \frac{1}{2}}\right)} \\
& +C v_{h}^{-\frac{3}{2}}\left\|u^{3}\right\|_{\widetilde{L}_{T}^{\infty}\left(B^{0, \frac{1}{2}}\right)}\left(\int_{0}^{t}\left\|\nabla_{h} u^{3}\right\|_{B^{0, \frac{1}{2}}}^{2}\left\|u^{h}\right\|_{B^{0, \frac{1}{2}}}^{2} d s\right)^{\frac{1}{2}}\left(1+\varepsilon_{2}^{-\frac{1}{2}}\left\|u^{h}\right\|_{\widetilde{L}_{t}^{\infty}\left(B^{0, \frac{1}{2}}\right)}^{\frac{1}{2}}\right) \\
& +C v_{h}^{-\frac{3}{2}}\left\|\nabla_{h} u^{3}\right\|_{\widetilde{L}_{t}^{2}\left(B^{0, \frac{1}{2}}\right)}\left\|u^{3}\right\|_{\widetilde{L}_{t}^{\infty}\left(B^{\left.0, \frac{1}{2}\right)}\right.} \varepsilon_{2}^{\frac{1}{2}}\left\|u^{h}\right\|_{\widetilde{L}_{t}^{\infty}\left(B^{0, \frac{1}{2}}\right)}^{\frac{1}{2}},
\end{aligned}
$$

where $t \in(0, T]$ and we choose $\varepsilon_{1}=\left\|u^{3}\right\|_{\widetilde{L}_{T}^{\infty}\left(B^{0, \frac{1}{2}}\right)}$. Similarly, we obtain the same estimate on $u_{n}^{2}$. Letting

$$
\varepsilon_{2}=\frac{\left\|u^{h}\right\|_{\widetilde{L}_{t}^{\infty}\left(B^{0, \frac{1}{2}}\right)}}{2 C v_{h}^{-3}\left\|\nabla_{h} u^{3}\right\|_{\widetilde{L}_{T}^{2}\left(B^{\left.0, \frac{1}{2}\right)}\right.}^{2}\left\|u^{3}\right\|_{\widetilde{L}_{T}^{\infty}\left(B^{0, \frac{1}{2}}\right)}^{2}},
$$

using Gronwall's inequality, we get

$$
\begin{aligned}
& \left\|u_{n}^{h}\right\|_{B^{0, \frac{1}{2}}(T)}^{2} \\
& \quad \leq\left(2 C_{0}\left\|u_{n}^{h}(0)\right\|_{B^{0, \frac{1}{2}}}^{2}+C v_{h}^{-1}\left\|u_{n}^{h}\right\|_{B^{0, \frac{1}{2}}(T)}^{3}\right) \exp \left\{C v_{h}^{-8}\left(\left\|u_{n}^{3}\right\|_{B^{0, \frac{1}{2}}(T)}+v_{h}\right)^{8}\right\} .
\end{aligned}
$$

Similarly, we obtain

$$
\left\|u_{n}^{3}\right\|_{B^{0, \frac{1}{2}(T)}} \leq 2 C_{0}\left\|u_{n}^{3}(0)\right\|_{B^{0, \frac{1}{2}}}+C v_{h}^{-\frac{1}{2}}\left\|u_{n}^{h}\right\|_{B^{0, \frac{1}{2}(T)}}^{\frac{1}{2}}\left\|u_{n}^{3}\right\|_{B^{0, \frac{1}{2}}(T)}+C v_{h}^{-\frac{1}{2}}\left\|u_{n}^{h}\right\|_{B^{0, \frac{1}{2}}(T)}^{\frac{3}{2}} .
$$

Then, we obtain

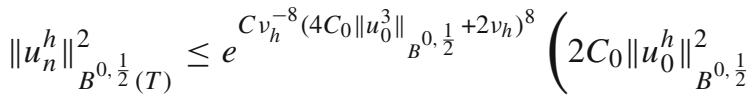

$$
\begin{aligned}
& \left.+C v_{h}^{-1}\left(4 C_{0}\left\|u_{0}^{h}\right\|_{B}^{2}{ }_{B, \frac{1}{2}}\right)^{\frac{3}{2}} e^{\frac{3}{2} C v_{h}^{-8}\left(4 C_{0}\left\|u_{0}^{3}\right\|_{B} 0, \frac{1}{2}+2 v_{h}\right)^{8}}\right)
\end{aligned}
$$

and

$$
\begin{aligned}
& \left\|u_{n}^{3}\right\|_{B^{0, \frac{1}{2}}(T)} \\
& \leq 2 C_{0}\left\|u_{0}^{3}\right\|_{B} 0, \frac{1}{2}+C v_{h}^{-\frac{1}{2}}\left(4 C_{0}\left\|u_{0}^{h}\right\|_{B}^{2}{ }_{B, \frac{1}{2}}\right)^{\frac{3}{4}} e^{\frac{3}{4} C v_{h}^{-8}\left(4 C_{0}\left\|u_{0}^{3}\right\|_{B}^{0, \frac{1}{2}}+2 v_{h}\right)^{8}} \\
& \quad+C v_{h}^{-\frac{1}{2}}\left(4 C_{0}\left\|u_{0}^{h}\right\|_{B}^{2}{ }^{0, \frac{1}{2}}\right)^{\frac{1}{4}} e^{\frac{1}{4} C v_{h}^{-8}\left(4 C_{0}\left\|u_{0}^{3}\right\|_{B}^{0, \frac{1}{2}}+2 v_{h}\right)^{8}}\left(4 C_{0}\left\|u_{0}^{3}\right\|_{B^{0, \frac{1}{2}}}+v_{h}\right),
\end{aligned}
$$

for all $T<T_{n}$, where

$$
\begin{gathered}
T_{n}:=\sup \left\{t>0 ;\left\|u_{n}^{h}\right\|_{B^{0, \frac{1}{2}(t)}}^{2} \leq 4 C_{0}\left\|u_{0}^{h}\right\|_{B^{0, \frac{1}{2}}}^{2} e^{C v_{h}^{-8}\left(4 C_{0}\left\|u_{0}^{3}\right\|_{B} 0, \frac{1}{2}+2 v_{h}\right)^{8}},\right. \\
\left.\left\|u_{n}^{3}\right\|_{B^{0, \frac{1}{2}(t)}} \leq 4 C_{0}\left\|u_{0}^{3}\right\|_{B^{0, \frac{1}{2}}}+v_{h}\right\},
\end{gathered}
$$


Then, if $u_{0}$ satisfies

$$
C_{1} v_{h}^{-1}\left\|u_{0}^{h}\right\|_{B}^{0, \frac{1}{2}} \exp \left\{C_{1}\left(v_{h}^{-1}\left\|u_{0}^{3}\right\|_{B^{0, \frac{1}{2}}}+1\right)^{8}\right\} \leq 1,
$$

where

$$
C_{1}=4{ }^{9} C^{2} C_{0}^{8}
$$

we get that for any $n$ and for any $T<T_{n}$,

$$
\left\|u_{n}^{h}\right\|_{B, \frac{1}{2}(T)} \leq \frac{5}{2} C_{0}\left\|u_{0}^{h}\right\|_{B}^{2}{ }^{0, \frac{1}{2}} e^{C v_{h}^{-8}\left(4 C_{0}\left\|u_{0}^{3}\right\|_{B} 0, \frac{1}{2}+2 v_{h}\right)^{8}}
$$

and

$$
\left\|u_{n}^{3}\right\|_{B, \frac{1}{2}(t)} \leq \frac{5}{2} C_{0}\left\|u_{0}^{3}\right\|_{B}{ }_{B, \frac{1}{2}}+\frac{1}{2} v_{h} .
$$

Thus, $T_{n}=+\infty$. Then, the existence follows from the classical compactness method.

Finally, we would like to thank Ping Zhang for pointing out this gap.

\section{Reference}

1. Zhang, T.: Global wellposed problem for the 3-D incompressible anisotropic Navier-Stokes equations in an anisotropic space. Commun. Math. Phys. 287, 211-224 (2009)

Communicated by P. Constantin 